\title{
A Interface Ensino-Extensão em Um Grupo de Gestante: Impactos na Formação Profissional
}

\section{The Teaching-Extension Interface in a Pregnant Group: Impacts on Training Professional}

\section{La Interfaz de Enseñanza y Extensión en un Grupo Embarazado: Impactos en la Formación Profesional}

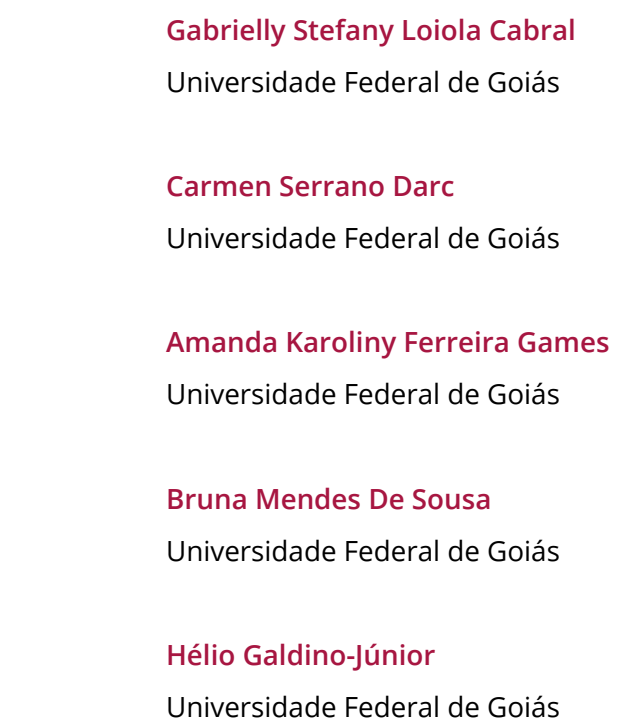

Resumo: O período gestacional é cercado por dúvidas e mitos e uma ferramenta que pode mitigar o problema nas unidades de saúde são os grupos. O objetivo deste trabalho é relatar a experiência de alunos do Programa de Educação Tutorial da Faculdade de Enfermagem em uma extensão para a implantação de um grupo de gestantes em uma unidade de saúde da família e apontar os impactos da ação na formação do enfermeiro. A metodologia constituiu de um relato de experiência da vivência na consolidação do grupo de gestantes. A percepção dos alunos foi verificada por 
meio da análise de conteúdo proposta por Bardin (2011). As gestantes reconheceram a importância do grupo, expondo dúvidas e mitos que são transmitidos de geração a geração. Os estudantes referiram que a atividade os aproximou da prática profissional, favorecendo o desenvolvimento de habilidades importantes para sua atuação profissional. Estes dados reforçam a importância do intercâmbio entre comunidade, serviço de saúde e universidade. Palavras-chave: Gestantes. Educação em Saúde. Programa de Educação Tutorial. Enfermagem. Extensão.

Abstract: The gestational period is surrounded by doubts and myths and a tool that can mitigate the problem in health units is the groups. The objective of this work is to report the experience of students of the Tutorial Education Program of the Faculty of Nursing in an extension for the implantation of a group of pregnant women in a family health unit and to point out the impacts of the action in the training of nurses. The methodology consisted of an experience report of the experience in the consolidation of the group of pregnant women. The students' perception was verified through the content analysis proposed by Bardin (2011). The pregnant women recognized the importance of the group, exposing doubts and myths that are transmitted from generation to generation. The students reported that the activity brought them closer to professional practice, favoring the development of important skills for their professional performance. These data reinforce the importance of exchanges between community, health service and university.

Keywords: Pregnant women. Health Education. Tutorial Education Program. Nursing. Extension.

Resumen: El período de gestación está rodeado de dudas y mitos y una herramienta que puede mitigar el problema en las unidades 
de salud son los grupos. El objetivo de este trabajo es informar la experiencia de los estudiantes del Programa de Educación Tutorial de la Facultad de Enfermería en una extensión para la implantación de un grupo de mujeres embarazadas en una unidad de salud familiar y señalar los impactos de la acción en la capacitación de enfermeras. La metodología consistió en un informe de experiencia de la experiencia en la consolidación del grupo de mujeres embarazadas. La percepción de los estudiantes se verificó mediante el análisis de contenido propuesto por Bardin (2011). Las mujeres embarazadas reconocieron la importancia del grupo, exponiendo dudas y mitos que se transmiten de generación en generación. Los estudiantes informaron que la actividad los acercó a la práctica profesional, favoreciendo el desarrollo de habilidades importantes para su desempeño profesional. Estos datos refuerzan la importancia de los intercambios entre la comunidad, el servicio de salud y la universidad.

Palabras clave: Mujeres embarazadas. Educación para la Salud. Tutorial del Programa de Educación. Enfermería. Extensión.

Data de submissão: 29/08/2020

Data de aprovação: 15/10/2020 
A Interface Ensino-Extensão em um Grupo de Gestante: Impactos

\section{Introdução}

O processo gestacional é um período vivenciado pela mulher onde ocorrem diversas mudanças fisiológicas e psicológicas, tendo variações entre gestantes e a cada processo gestacional. Esta fase é carregada de crenças e mitos que perpetuam por gerações e que vão construindo uma representação da gestação (MONTEIRO et al., 2011), e que com o avanço do conhecimento científico precisam ser desmistificadas, possibilitando transformações na vida dessas gestantes.

Um exemplo de mito na gestação é a prática de exercícios físicos durante a gravidez, Gouveia et al. (2007) em sua pesquisa identificou uma alta porcentagem de gestantes que abandonaram a prática de exercícios por causa da gestação. No entanto, recomenda-se que exercícios devem ser mantidos durante toda a gestação, visto que dentre seus benefícios estão à redução do edema, melhora da circulação sanguínea, ampliação do equilíbrio muscular, promove alívio dos desconfortos intestinais (incluindo a obstipação), diminui câimbras, fortalece a musculatura abdominal e facilita na recuperação no pós-parto (SURITA, NASCIMENTO e SILVA, 2014). Porém, muitos mitos são disseminados em relação a essa temática, privando as gestantes de adotarem a prática frequente de exercícios físicos.

Outros mitos envolvem temas como alimentação e seus reflexos na amamentação, sono, mudanças físicas e fisiológicas na mulher e no feto. Muitas gestantes, apesar de saberem que são mitos ainda os praticam, devido não receberem orientações esclarecedoras e acabam se apoiando na família, principal propagadora dos mesmos (SILVA et al., 2015). Visando a promoção da saúde da população, uma técnica bastante utilizada na atenção básica é 
A Interface Ensino-Extensão em um Grupo de Gestante: Impactos na Formação Profissional

Gabrielly Stefany Loiola Cabral • Carmen Serrano Darc, et al...

a realização de grupos, dessa forma grupos de gestantes podem contribuir para aumentar o conhecimento das pacientes neste processo repleto de mudanças (LIMA et al., 2018; WILD, SILVEIRA e FAVERO, 2014). Além disso, a promoção da saúde é um importante pilar na atividade profissional do enfermeiro, que deve atuar estimulando a adoção de estratégias de autocuidado, promovendo autonomia e qualidade de vida (MACENO e HEIDEMANN, 2016).

Desta forma, trabalhar em grupo permite a criação de vínculos entre os usuários e os profissionais da saúde, relações de interdependência e existência de afetos entre os participantes e com isso permite que os integrantes estejam à vontade para esclarecer as dúvidas referente a qualquer tema, possibilitando também a desconstrução de diversos mitos (LIMA et al., 2018; WILD, SILVEIRA e FAVERO, 2014). Além disso, o grupo de gestantes é uma estratégia importante, tendo em vista a contribuição das informações e trocas de experiências nele compartilhadas, permitindo uma vivência mais segura e orientada das participantes em suas gestações, bem como uma assistência de qualidade e humanizada ao binômio mãe-filho (HENRIQUES et al., 2015).

Apesar de estudos apontarem o impacto positivo no incremento do conhecimento da comunidade com a utilização de grupos na promoção da saúde e prevenção de agravos (MENEZES E AVELINO, 2016) esta tecnologia leve e de baixo custo na atenção à saúde humana, é pouco empregada, principalmente por falta de habilidade na técnica ou descrença na sua eficácia, ficando as atividades de educação e prevenção com menos destaque no tempo de atendimento dos profissionais da atenção básica (BRITO, MENDES, e NETO, 2018). Várias unidades de atenção à saúde da família não possuem grupos de gestantes, ou não estão em funcionamento, seja por desvalorização dessa ferramenta ou falta de habilidade dos profissionais em utilizá-la, o que evidencia uma oportuni- 
A Interface Ensino-Extensão em um Grupo de Gestante: Impactos

na Formação Profissional

Gabrielly Stefany Loiola Cabral • Carmen Serrano Darc, et al...

dade de intervenção e colaboração com a sociedade por meio da extensão universitária.

A extensão universitária é considerada como um importante elo entre a comunidade e universidade. Assume um importante papel no desenvolvimento crítico e reflexivo do estudante, atuando como uma promotora na troca de saberes populares e científicos e como difusor da educação em saúde no âmbito universitário e comunitário (SIQUEIRA et al., 2017; FERNANDES et al., 2013). Assim, a comunidade se beneficia do conhecimento produzido na universidade e os alunos vão adquirindo habilidades e vivências que refletirão na sua formação profissional. O Programa de Educação Tutorial (PET) tem como um dos seus pressupostos, ampliar a formação acadêmica, promovendo experiencias extracurriculares que culminam na ampliação de habilidades técnicas e interpessoais do graduando baseado na indissociabilidade do ensino, pesquisa e extensão (MINISTÉRIO DA EDUCAÇÃO, 2006).

Assim, o grupo PET enfermagem ao vislumbrar a carência de educação em saúde à população de gestantes de uma unidade de saúde da família do município de Goiânia, propôs uma ação de extensão na unidade com a finalidade de implantar e consolidar o grupo de gestantes na Unidade. Assim, este artigo tem como objetivo relatar a experiência do grupo PET enfermagem da Universidade Federal de Goiás, na implantação e consolidação de um grupo de gestantes e identificar os reflexos desta atividade de extensão na formação dos alunos participantes do projeto.

\section{Materiais e Métodos}

Trata-se de um relato de experiência baseado na implantação e consolidação de um grupo de gestantes em uma Unidade Básica de Saúde (UBS) no município de Goiânia-GO, por alunos 
A Interface Ensino-Extensão em um Grupo de Gestante: Impactos na Formação Profissional

Gabrielly Stefany Loiola Cabral • Carmen Serrano Darc, et al...

do Programa de Educação Tutorial (PET) da Faculdade de Enfermagem da Universidade Federal de Goiás (FEN/UFG), que ocorreu entre agosto de 2018 a dezembro de 2019. Inicialmente, os alunos ao realizarem estágio curricular na unidade, perceberam que não havia atividade de educação em saúde voltada para as gestantes. A questão foi levada para o grupo de tutoria, que decidiu promover uma ação de extensão que fosse contínua para a instalação e manutenção do grupo de gestantes na unidade.

Estabelecidas as parcerias entre o grupo PET e os enfermeiros coordenadores das equipes de saúde da família, realizaram-se reuniões de planejamento, baseadas no arco de Magarez (BERBEL, 2012), seguindo as seguintes etapas: observação da realidade, levantamento de pontos-chave, teorização, hipótese de solução e aplicação a realidade.

Baseado na observação da realidade e no levantamento dos postos-chaves, foi construído um planejamento, o qual foi aprovado pelo enfermeiro da unidade. O plano foi centrado nos temas: alterações morfofisiológicas no corpo da mulher, alimentação saudável, sexualidade durante o período gestacional, tipos de parto, aleitamento materno, cuidados com as mamas e aspectos psicológicos do puerpério. Para a aplicação dos temas, os alunos se aprofundaram no conteúdo e se apropriaram de técnicas grupais, com foco nas metodologias ativas.

Para conhecer os reflexos da atividade na formação dos petianos, realizou-se uma pesquisa de abordagem qualitativa. Os alunos que participaram de todas as etapas do projeto foram convidados para uma entrevista, respondendo a seguinte pergunta " $O$ que foi para você participar do grupo de gestantes?". Dos seis que eram elegíveis para a entrevista, quatro participaram. As entrevistas foram gravadas, e posteriormente transcritas e analisadas segundo o método de análise de conteúdo proposto por Bardin 
A Interface Ensino-Extensão em um Grupo de Gestante: Impactos na Formação Profissional

Gabrielly Stefany Loiola Cabral • Carmen Serrano Darc, et al...

(2011), tal método se organiza em três polos cronológicos: pré-análise, exploração do material e tratamento dos resultados. Esta análise consiste em identificar núcleos de sentido expressos durante a entrevista, em que a sua presença ou frequência signifique algo para o estudo. Para operacionalizar a análise de conteúdo foram percorridas as etapas de pré-análise, com leitura flutuante, seguida de leitura exaustiva das entrevistas buscando apreender as ideias centrais das falas, tratamento dos resultados obtidos em que as ideias centrais foram agrupadas originando as categorias e por último, a interpretação dos dados dialogando com a literatura disponível. Para apresentação dos resultados, os fragmentos das falas ilustrativos das categorias foram identificados pela letra " $\mathrm{P}$ " de "Petiano", seguidos de algarismo arábico segundo a ordem cronológica de realização da entrevista.

O projeto de extensão estava cadastrado ao Sistema Integrado de Gestão de Atividades Acadêmicas da Universidade Federal de Goiás (Sigaa/UFG), além de estar contido no planejamento das atividades anuais realizadas pelo PET Enfermagem/UFG. Além disto, foi aprovado no comitê de ética em pesquisa do Hospital das Clínicas UFG, sob parecer $n^{\circ} 2.403 .287$, projeto de pesquisa que respaldou a coleta de dados dos alunos participantes da ação.

\section{Resultados e Discussão}

Os encontros ocorreram mensalmente, possuindo uma hora de duração, abordando um tema em cada reunião. Realizaram-se técnicas de acolhimento e relaxamento para construção de vínculo com o grupo e melhor desenvolvimento da temática abordada. Os universitários expunham o tema, de forma expositiva-dialogada, utilizando técnicas grupais e rodas de conversa, permitindo às usuárias levantarem dúvidas ou vivências pessoais. Durante 
A Interface Ensino-Extensão em um Grupo de Gestante: Impactos na Formação Profissional

Gabrielly Stefany Loiola Cabral • Carmen Serrano Darc, et al...

alguns encontros, participaram também pais e puérperas, proporcionando ampliação da abordagem. As gestantes foram bastante colaborativas, expondo suas crenças e mitos sobre o período gestacional, parto e puerpério, as quais foram trabalhadas à medida que as questões emergiam no grupo, ou propostas para serem abordadas em um novo encontro.

$\mathrm{Na}$ avaliação do grupo, as gestantes destacaram o tema "Amamentação", sendo um momento temido e importante de ser trabalhado, assim como mostrado em outros estudos, (CUNHA, SANTOS, e GONÇALVES, 2012), reafirmando a importância do grupo para o compartilhamento de experiências e a resolução de dúvidas. $O$ encontro da Universidade com a comunidade no serviço de saúde, permitiu às gestantes esclarecer dúvidas e compartilhar experiências com outras usuárias. Dos quatro principais temas emergiam mitos e crenças que foram tabulados e apresentados no quadro 1.

\begin{tabular}{|c|c|}
\hline Temas & Mitos e Crenças \\
\hline $\begin{array}{l}\text { Alterações morfofisio- } \\
\text { lógicas no corpo da } \\
\text { mulher. }\end{array}$ & $\begin{array}{l}\text { “Toda gestante sente náuseas"; “Barriga pontuda é } \\
\text { menino, barriga arredondada é menina”; “Gestante } \\
\text { não deve praticar exercício físico". }\end{array}$ \\
\hline Alimentação saudável & $\begin{array}{l}\text { “Quanto maior o peso da mãe, maior será o bebê”; } \\
\text { "Gestantes devem restringir carboidratos e gor- } \\
\text { duras, e comer mais proteínas"; “O consumo de } \\
\text { sal não influencia a gravidez"; “Grávida tem que } \\
\text { comer por dois"; “O consumo de queijo na gestação } \\
\text { é contraindicado"; “Os desejos alimentares não } \\
\text { atendidos fazem com que o bebê nasça com algum } \\
\text { problema"; “O consumo de leite na gestação faz mal } \\
\text { para o bebê"; “Beber } 1 \text { taça de vinho não faz mal ao } \\
\text { bebê". }\end{array}$ \\
\hline
\end{tabular}


A Interface Ensino-Extensão em um Grupo de Gestante: Impactos na Formação Profissional

Gabrielly Stefany Loiola Cabral • Carmen Serrano Darc, et al...

\begin{tabular}{l|l|}
\hline $\begin{array}{l}\text { Aleitamento materno } \\
\text { e cuidados com as } \\
\text { mamas. }\end{array}$ & $\begin{array}{l}\text { "Cerveja preta ajuda a aumentar a produção de } \\
\text { leite"; “O aleitamento prejudica o seio da mulher"; } \\
\text { "Devem-se limpar as mamas a cada mamada". }\end{array}$ \\
\hline $\begin{array}{l}\text { Tipos de parto e sexu- } \\
\text { alidade }\end{array}$ & $\begin{array}{l}\text { "Uma mãe que fez cesárea não pode ter parto } \\
\text { normal na próxima gestação"; “O parto normal } \\
\text { sempre vem acompanhado da episiotomia"; “O } \\
\text { parto normal deixa o canal vaginal mais largo e, por } \\
\text { isso, demora mais para as mães retomarem a vida } \\
\text { sexual"; “Mulheres com quadris largos têm mais } \\
\text { facilidade para ter o parto normal”. }\end{array}$ \\
\hline
\end{tabular}

Quadro 1: Principais mitos/crenças das gestantes sobre os temas. (Goiânia, 2020).

A gestação é um período importante para realização de ações educativas como esta, por propiciar um intercâmbio de vivências e conhecimentos. Assim, o grupo de gestantes pode ser considerado uma forma de promover a compreensão do processo de gestação. Diferentes experiências podem ser compartilhadas entre as mulheres e os profissionais de saúde (HENRIQUES et al, 2015). Pode-se observar uma gama de mitos, que foram desvelados pela atividade de extensão, promovendo o conhecimento e a autonomia da mulher nesse importante período gravídico-puerperal. Principalmente mitos relativos ao tipo de parto, visto que no Brasil ainda se pratica altos índices de parto cesáreo (ZAIDEN et al., 2020). Estudos em outras regiões do país também mostram a efetividade de grupos de gestantes na adoção de hábitos saudáveis proveniente do conhecimento agregado com a utilização dos grupos como estratégia de educação em saúde (CARDOSO et al 2019; RICCl et al, 2019).

No que diz respeito à percepção dos petianos com a vivência no grupo de gestantes, emergiram com a análise das falas duas categorias principais, sendo elas intituladas de: "troca de conhecimentos" e "habilidades intrínsecas a enfermagem". 
A Interface Ensino-Extensão em um Grupo de Gestante: Impactos

na Formação Profissional

Gabrielly Stefany Loiola Cabral • Carmen Serrano Darc, et al...

\section{Categoria "Habilidades intrínsecas a enfermagem"}

Nesta categoria, os alunos do PET evidenciaram a importância de participarem de ações educativas pelo fato de os permitirem desenvolver habilidades que serão fundamentais durante sua atuação como futuros profissionais de enfermagem.

“Então, dentro desse grupo eu desenvolvi habilidades como: comunicação mais efetiva, trabalhar a liderança, trabalhar em grupo, conduzir um grupo, e compartilhamento de experiências com uma população mais específica" (P1).

"A experiencia fica pra depois você usar em orientação quando estiver fazendo uma consulta pré-natal, ou quando tiver fazendo outros grupos de gestantes com outras gestantes em sua vida profissional" (P2).

"Me permitiu aprimorar técnicas de comunicação, principalmente a transmissão de conhecimento de uma forma que ficasse clara para aquele público onde o grau de escolaridade era diverso. E assim, terei que fazer isso durante a minha vida profissional né" (P3).

"Para formar o grupo precisava trabalhar e desenvolver a comunicação, desenvoltura em público, planejamento dos encontros, estudar os diversos assuntos que envolvem uma gestação, criar vínculo com as gestantes da unidade, auxiliando no processo de pré-natal o que evidencia a importância desses grupos para a formação do petiano/profissional" (P4).

Dentre as habilidades citadas pelos alunos, que foram desenvolvidas na atividade de extensão, estão a liderança e comunicação. No contexto da enfermagem a liderança é reconhecidamente uma habilidade essencial para a gestão do cuidado humano (ALMEIDA de MOURA et al., 2019) e estudos apontam que essas características são pouco desenvolvidas na graduação em enfermagem (LEITE SIQUEIRA et al., 2019). Das habilidades interpessoais consideradas 
A Interface Ensino-Extensão em um Grupo de Gestante: Impactos

na Formação Profissional

Gabrielly Stefany Loiola Cabral • Carmen Serrano Darc, et al...

essenciais ao mercado de trabalho (HURRELL, 2016; SUCCI, 2019) as principais foram compatíveis com as relatadas pelos alunos como sendo fruto da sua participação na ação de extensão: Comunicação, trabalho em equipe, gestão e liderança e tomada de decisão.

Os acadêmicos de enfermagem ao longo da graduação vivenciam vários momentos de práticas, porém nem sempre são suficientes, sendo assim importante a participação destes em nas ações extensionistas que propiciam o desenvolvimento de habilidades técnicas e interpessoais, uma vez que proporciona aos alunos vivências na comunidade, que muitas vezes não são oportunizadas apenas na graduação e ainda por meio da extensão podem transformar a vida das pessoas com o conhecimento e compartilhamento das melhores evidências da sua área de atuação profissional.

12

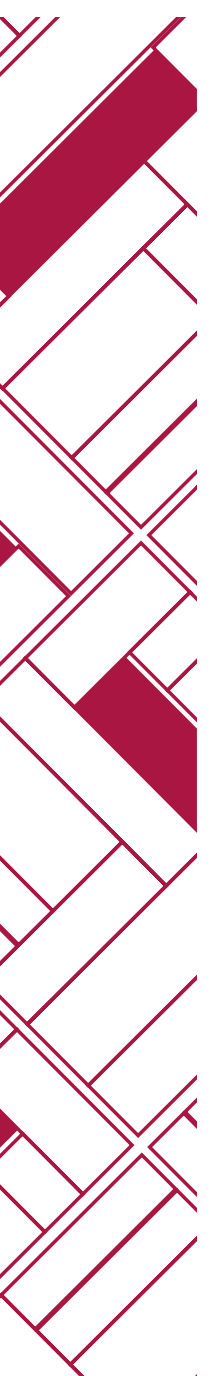

\section{Conclusões}

A vivência com o grupo de gestantes permitiu o esclarecimento de dúvidas e desmistificação de mitos apresentados pelas participantes, havendo, portanto, a promoção da saúde da mulher no período gravídico-puerperal. Possibilitou ainda, uma experiência de integração entre acadêmicos e usuárias da atenção básica bem como o estreitamento das relações entre universidade e o serviço de saúde. Além disso, permitiu o aprimoramento de habilidades de coordenação de grupo por parte dos discentes integrantes do grupo PET, e ainda impulsionou a continuidade do grupo de gestantes na unidade, que ainda se encontra ativo atualmente.

Contudo, os impactos do grupo de gestantes não se limitaram às gestantes. Participar do projeto, também refletiu diretamente na formação acadêmica dos estudantes. O contato com um grupo específico, com realidades e desafios distintos proporcionou aos alunos uma ampliação de conhecimentos e experiências e trouxe 
A Interface Ensino-Extensão em um Grupo de Gestante: Impactos na Formação Profissional

Gabrielly Stefany Loiola Cabral • Carmen Serrano Darc, et al...

benefícios não somente à vida acadêmica, mas, também, para o âmbito profissional. Esse tipo de atividade proporcionou ao aluno desenvolver habilidades de trabalho em equipe, liderança, comunicação, autoconhecimento, entre outras competências essenciais na vida profissional do enfermeiro.

Vivenciasse uma experiência de trabalhar no grupo de gestantes, constituiu-se em um espaço de socialização de trocas e conhecimentos, sendo uma oportunidade para os petianos transmitirem e adquirirem o alicerce que iram utilizar posteriormente. Além disso, permitiu o relacionamento de futuros profissionais de saúde com a prática, o que possibilita um melhor enfrentamento da realidade que irá conviver.

\section{Referências}

ALMEIDA DE MOURA, ANDRÉ, ET AL. LIDERANÇA CARISMÁTICA ENTRE OS PROFISSionais de enfermagem: revisão integrativa. Revista Brasileira de EnfermaGEM, V. 72, 2019.

Bardan, Laurence. Análise de conteúdo. São Paulo: Almedina Brasil. 2011.

Benito, Gladys Amelia Vélez et al. Desenvolvimento de competênCias gerais duRante o estágio supervisionado. Revista Brasileira de Enfermagem, v. 65, N. 1, P. 172-178, 2012.

Berbel, Neusi Aparecida Navas. A metodologia da problematização com o Arco de Maguerez: uma reflexÃo teórico-epistemológica. ScIELO-EDUEL, 2012. Brito, Geraldo Eduardo Guedes de; MENDES, Antonio da Cruz GouVeia; SAntos neto, Pedro Miguel dos. O trabalho na estratégia saúde da família e a PERSISTÊNCIA das práticas CURATIVISTAS. Trabalho, Educação e Saúde, v. 16, N. 3, P. 975-995, 2018. 
A Interface Ensino-Extensão em um Grupo de Gestante: Impactos na Formação Profissional

Gabrielly Stefany Loiola Cabral • Carmen Serrano Darc, et al...

CARDOSO, Soraya Lopes et AL. Ações de PROMoção para SAÚde DA GESTANTE com Ênfase no pré-natal. Revista Interfaces: Saúde, Humanas e TecnoloGIA, V. 7, N. 1, P. 180-186, 2019.

DA CUNHA, Ana Cristina Barros; SANTOS, Carmelita; GONÇALVES, Raquel Menezes. Concepções Sobre maternidade, parto e amamentação em grupo de gestantes. Arquivos Brasileiros de Psicologia, v. 64, n. 1, P. 139-155, 2012.

DA SiLVA, Thamiles Sena et Al. A extensÃo universitáRIA e a PreVenção da VlolênCIA obstétrica. Revista CiênCia em Extensão, v. 13, N. 1, P. 176-189, 2017.

DAMASCENO, RenATA FIÚZA; BRUNÓRIO, LUdIMILA; ANDRADE, Maria BetÂNIA TINTI DE. O PRograma de EDUCAÇÃO TUTORIAL-PET-SOB A ÓtICA DOS INICIantes. Revista Mineira de Enfermagem, v. 10, N. 2, P. 160-165, 2006.

De MeneZeS, Parreiras; KIEFER, Kênia; ROBerto aVElinO, Patrick. Grupos operativos na Atenção Primária À Saúde como prática de discussão e educação: uma revisão. Cadernos Saúde Coletiva, v. 24, N. 1, 2016.

FERNANDES, Josicelia DumÊT et AL. AderênCIA de CURSOS de GRAduação em ENFERMAGEM ÀS DIRETRIZES CURRICULARES NACIONAIS NA PERSPECTIVA DO SISTEMA único de saúde. Escola Anna Nery Revista de Enfermagem, v. 17, N. 1, P. 82-89, 2013.

GOUVEIA, Raquel et al. Gravidez e eXercício físico: mitos, eVIdênCias e recomendações. Acta Médica Portuguesa, P. 209-214, 2007.

HENRIQUES, Amanda Haissa Barros et al. Grupo de Gestantes: conTRIBUIÇÕES E POTENCIALIDADES NA COMPLEMENTARIDADE DA ASSISTÊNCIA PRÉ-NATAL. Revista Brasileira em Promoção da Saúde, v. 28, n. 1, P. 23-31, 2015. HURRELL, Scott A. Rethinking the soft SkILls deficit blame game: EmployERS, SKILLS WITHDRAWAL AND the REPORTING Of SOFt SKILLS GaPS. Human ReLATIONS, v. 69, N. 3, P. 605-628, 2016. 
A Interface Ensino-Extensão em um Grupo de Gestante: Impactos na Formação Profissional

Gabrielly Stefany Loiola Cabral • Carmen Serrano Darc, et al...

Leite SiQueIRA, Cibele et al. ConheCimento de enfermeiros responsáveis

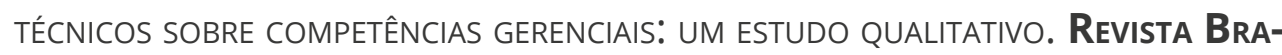
Sileira de Enfermagem, v. 72, N. 1, 2019.

LiMA, Margarete Maria de et al. Contribuição da extensão em um Grupo de GESTANTES E CASAIS GRÁVIDOS PARA A FORMAÇÃo do ENFERMEIRO. Escola AnNA NerY, v. 22, N. 4, 2018.

maCeno, Priscila Rosa; HeIDEMANN, Ivonete Teresinha Schulter Buss. UnVeiling the actions of nURSES IN PRIMARY health CARE GROUPS. Texto \& Contexto-Enfermagem, v. 25, N. 4, 2016.

MiNistério da eduCAÇÃo. Programa de Educação Tutorial-PET: Manual de Orientações Básicas. Brasília. Dez, 2006.

MONTEIRO, Aldora et al. A visão da mulher na ANTROPOlogia: Mitos DA CRIAÇão e CRençAS em relaçÃo À GRavidez. Millenium-Journal of Education, Technologies, and Health, N. 30, P. 38-50, 2016.

RICCl, Ana PatríCia et al. Infecções SeXUalmente transmissíveIS na GestaÇÃo: EDUCAÇÃO EM SAÚDE COMO ESTRATÉGIA DE PREVENÇÃO NA ATENÇÃO BÁSICA/SEXUALLY TRANSMITTED INFECTIONS DURING PREGNANCY: HEALTH EDUCATION AS A PREVENTION strategy in primary care. Brazilian Journal of Health Review, v. 2, N. 1, P. 565-570, 2019.

DA SilVA, Luzenilda Sabina; De BORBA PeSSOA, Franciele; PeSSOA, Douglas Tadeu Cardoso. AnÁlise das mudançAS FIsIOlÓgICAS DURANTE A gestação: desvendando mitos. Revista Eletrônica Faculdade Montes BeLoS, v. 8, N. 2, 2015.

DA SilVA, Valdenir Almeida; DA CRUZ, Jefferson Bruno Ribeiro Lima; DE CAMARGO, Climene Laura. O programa de educação tutorial (PET) como instrumento pedagógico para os alunos de enfermagem. Revista Baiana de Enfermagem, v. 22, N. 1, 2008. 
A Interface Ensino-Extensão em um Grupo de Gestante: Impactos na Formação Profissional

Gabrielly Stefany Loiola Cabral • Carmen Serrano Darc, et al...

SiQUelRA, Samylla Maira Costa et al. Atividades extensionistas, promoção DA SAÚDE E DESENVOLVIMENTO SUSTENTÁVEL: EXPERIÊNCIA DE UM GRUPO DE PESQUISA em enfermagem. Escola Anna Nery Revista de Enfermagem, v. 21, n. 1, p. 1-7, 2017.

SUCCl, Chiara. Are you ready to find a job? Ranking of a list of Soft Skills to enhance graduates' employability. International Journal of Human

Resources Development and Management, v. 19, N. 3, P. 281-297, 2019.

SuRItA, Fernanda Garanhani et al. Exercício físico e gestação. Revista BRASILEIRA DE GINECOLOGIA E OBSTETRÍCIA, v. 36, N. 12, P.531-534. 2014 FERNANDES WILD, CAmila et Al. Ações educativas com o grupo de GesTANTES USUÁRIAS dA ATENÇÃO BÁSICA: RELATO DE EXPERIÊNCIA. JouRNAL OF NuRSINg UFPE/Revista de Enfermagem UFPE, v. 9, N. 10, 2015.

ZAIDEN, LAURA ET AL. INFLUÊNCIA DAS CARACTERÍSTICAS HOSPITALARES NA REALização de cesárea eletiva na Região Sudeste do Brasil. Cadernos de Saúde Pública, v. 36, P. E00218218, 2020. 